\title{
Números de Stirling do primeiro tipo
}

\author{
Gabriel F. Pinheiro ${ }^{1}$ (D) Irene M. Craveiro(D) Naiguiel A. da Silva ${ }^{2}$ (D)
}

\begin{abstract}
Resumo
Neste trabalho investigamos as conexões existentes entre os números de Stirling do primeiro tipo e os grupos de permutações de n elementos. Apresentamos resultados conhecidos no intuito de elucidar a relação entre os números de Stirling e os conteúdos ensinados no Ensino Médio.

Palavras-chave: Números de Stirling; Ensino Médio.

\section{Abstract}

In this work we investigate the existing connections between the Stirling numbers of the first kind and the permutations groups on $\mathrm{n}$ elements. We present known results order to elucidate the relationship between Stirling numbers and contents taught in High School.
\end{abstract}

Keywords: Stirling numbers; High School.

\section{Introdução}

Números de Stirling demonstram ser uma ferramenta útil para lidar com diversos problemas combinatórios. Dessa forma, desenvolvemos diversas propriedades para os números de Stirling do primeiro tipo, apresentando uma prova algébrica ou combinatória. Os números de Stirling do primeiro tipo podem ser definidos por meio de permutações de um conjunto de $n$ objetos, $I_{n}=\left\{c_{1}, c_{2}, \ldots, c_{n}\right\}$. Sem perda de generalidade vamos supor que $I_{n}=\{1,2, \ldots, n\}$. Em seguida, estabelecemos uma recorrência para os números de Stirling do primeiro tipo. A partir dessa relação de recorrência instituimos a função geradora ordinária, ou seja, um polinômio na indeterminada x, cujos coeficientes das potências de x são números de Stirling do primeiro tipo.

A recorrência dos números de Stirling do primeiro tipo também permite dispor esses números na forma triangular; esse triângulo formado é similar ao Triângulo de Pascal formado pelos coeficientes binomiais. Os coeficientes binomiais podem ser interpretados combinatoriamente como o número de subconjuntos com $\mathrm{k}$ elementos de um conjunto com n objetos, enquanto que o número de Stirling do primeiro tipo pode ser interpretado como a quantidade de permutações de um conjunto de $\mathrm{n}$ elementos que se decompõe em $\mathrm{k}$ ciclos.

\footnotetext{
${ }^{1}$ Agradeço à UFGD pela concessão de bolsa de Iniciação Científica.

${ }^{2}$ Apoio Capes.
} 


\section{O Grupo de Permutações de n objetos}

Nesta seção apresentamos diversos conceitos e resultados referentes ao grupo de permutações de $n$ objetos. Os resultados que validamos agora seguem de [5].

Definição 1. Um grupo consiste de um conjunto não vazio G munido de uma operação indicada por * (isto é, uma regra em que cada par ( $\mathrm{x}, \mathrm{y})$ de $\mathrm{G}$ associa um terceiro elemento de $\mathrm{G}$, indicado por $\mathrm{x} * \mathrm{y})$ satisfazendo as seguintes propriedades:

- associatividade: dados $\mathrm{x}, \mathrm{y}, \mathrm{z} \in \mathrm{G}$ vale $\mathrm{x} *(\mathrm{y} * \mathrm{z})=(\mathrm{x} * \mathrm{y}) * \mathrm{z}$;

- existência do elemento neutro: para todo $\mathrm{x} \in \mathrm{G}$, existe e $\in \mathrm{G}$ tal que $\mathrm{x} * \mathrm{e}=\mathrm{x}$;

- existência do elemento inverso: para cada $\mathrm{x} \in \mathrm{G}$, existe $\mathrm{y} \in \mathrm{G}$ tal que $\mathrm{x} * \mathrm{y}=\mathrm{e}$.

Se a operação $*$ satisfaz a propriedade comutativa (isto é, para todos $\mathrm{x}, \mathrm{y} \in \mathrm{G}$ vale $\mathrm{x} * \mathrm{y}=\mathrm{y} * \mathrm{x}$ ) dizemos que $\mathrm{G}$ é um grupo comutativo ou abeliano.

Observação 1. Os elementos neutro e inverso são únicos.

Exemplo 1. Considere o conjunto dos números inteiros $\mathbb{Z}$ com a operação usual de adição. Sabemos que dados $\mathrm{x}, \mathrm{y} \in \mathbb{Z}$ temos que $\mathrm{x}+\mathrm{y} \in \mathbb{Z}$, logo, a operação de adição é fechada em $\mathbb{Z}$. Segue assim que, em $\mathbb{Z}$, vale a associatividade. Além disso, e = 0 é o elemento neutro da adição; de fato, dado $\mathrm{x} \in \mathbb{Z}$ temos que $\mathrm{x}+0=\mathrm{x}$. Por fim, para cada $\mathrm{x} \in \mathbb{Z}$ existe $-\mathrm{x} \in \mathbb{Z}$ tal que $\mathrm{x}+(-\mathrm{x})=0$. Conclui-se dessa forma que $\mathbb{Z}$ com a operação de adição usual é um grupo.

Definição 2. Uma permutação de um conjunto não vazio X é uma bijeção de X em X. Denotamos por $\mathrm{S}(\mathrm{X})$ o conjunto de todas as permutações de $\mathrm{X}$.

Exemplo 2. Considere X o conjunto formado pelos quatro ases de um baralho comum, disposto em qualquer ordem, como mostra a figura abaixo:

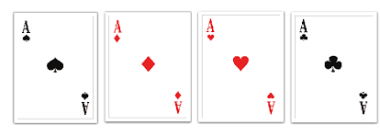

Figura 1: Sequência de ases

É natural supor que a ordem estabelecida para se dispor as cartas é arbitrária e que, além disso, a sequência dada na Figura 1 não é a única iniciada pelo ás de espadas. Assim caso se deseje começar a sequência pelo ás de ouros em vez de iniciar pelo ás de espadas, bastaria posicionar a carta citada na primeira posição e dispor as demais em uma ordem qualquer. Desse modo, se quisermos obter uma certa sequência dos quatro ases a partir do embaralhamento de uma sequência previamente estipulada, já não poderemos arbitrar a ordem em que colocaremos cada carta, uma vez que, a partir da sequência inicial, cada embaralhamento corresponderá a uma determinada ordem das cartas

Exemplo 3. Considere $X=\mathbb{R}$ o conjunto dos números reais. Observe que a função $f: \mathbb{R} \rightarrow \mathbb{R}$ dada por $\mathrm{f}(\mathrm{x})=\mathrm{x}$, para todo $\mathrm{x} \in \mathbb{R}$, é uma permutação de $\mathbb{R}$ em $\mathbb{R}$. Para isso, basta mostrarmos que $\mathrm{f}$ é uma bijeção. Note que, dados $\mathrm{x}_{1} \neq \mathrm{x}_{2}$ em $\mathbb{R}$ implica que $\mathrm{f}\left(\mathrm{x}_{1}\right)=\mathrm{x}_{1} \neq \mathrm{x}_{2}=\mathrm{f}\left(\mathrm{x}_{2}\right)$, logo, $\mathrm{f}$ é injetora. Além disso, dado $\mathrm{y} \in \mathbb{R}$, existe $\mathrm{x}=\mathrm{y}$ em $\mathbb{R}$ tal que $\mathrm{f}(\mathrm{x})=\mathrm{f}(\mathrm{y})=\mathrm{y}$, ou seja, $\mathrm{f}$ é sobrejetora. Potanto, f é bijetora e, consequentemente, uma permutação. 
Observação 2. Note que $\mathrm{S}(\mathrm{X})$ munido da operação de composição de funções é um grupo, chamado de Grupo das Permutações de X. De fato, a composição de funções é associativa; além disso, como todo elemento de $\mathrm{S}(\mathrm{X})$ é uma bijeção, existe o elemento inverso e, por fim, o elemento neutro é dado pela função $\mathrm{I}: \mathbb{X} \rightarrow \mathbb{X}$ dada por $\mathrm{I}(\mathrm{x})=\mathrm{x}$, para todo $\mathrm{x} \in \mathrm{X}$. Quando $\mathrm{X}=\{1,2, \ldots, \mathrm{n}\}$ denotamos $\mathrm{S}(\mathrm{X})$ por $\mathrm{S}_{\mathrm{n}}$ e o chamamos de Grupo Simétrico de Grau n. Observe que a ordem (quantidade de elementos do conjunto) de $\mathrm{S}_{\mathrm{n}}$ é $\mathrm{n}$ !. De fato, segue da análise combinatória que o número de permutações possíveis para um conjunto com n elementos é $\mathrm{P}_{n}=n$ !.

Exemplo 4. Seja $X=\{1,2,3\}$. Temos que a ordem de $S_{3}$ é $3 !=6$. De fato, podemos montar as seguintes sequências com os elementos de X: $(1,2,3),(1,3,2),(2,1,3),(2,3,1),(3,1,2),(3,2,1)$.

Observação 3. Seja $\mathrm{X}=\{1,2, \ldots, \mathrm{n}\}$ e f uma permutação de $\mathrm{X}$. Podemos denotar $\mathrm{f}$ de $\mathrm{S}_{\mathrm{n}}$ na forma de diagrama, $\left(\begin{array}{ccccc}1 & 2 & 3 & \ldots & \mathrm{n} \\ \mathrm{f}(1) & \mathrm{f}(2) & \mathrm{f}(3) & \ldots & \mathrm{f}(\mathrm{n})\end{array}\right)$, em que a primeira linha representa os elementos do conjunto X e a segunda linha representa a permutação feita com tais componentes. A partir disso, vamos elencar todos os elementos de $\mathrm{S}_{4}$ na forma de diagrama:

$$
\begin{aligned}
& \left(\begin{array}{llll}
1 & 2 & 3 & 4 \\
1 & 2 & 3 & 4
\end{array}\right),\left(\begin{array}{llll}
1 & 2 & 3 & 4 \\
1 & 2 & 4 & 3
\end{array}\right),\left(\begin{array}{llll}
1 & 2 & 3 & 4 \\
1 & 4 & 2 & 3
\end{array}\right),\left(\begin{array}{llll}
1 & 2 & 3 & 4 \\
1 & 4 & 3 & 2
\end{array}\right) \\
& \left(\begin{array}{llll}
1 & 2 & 3 & 4 \\
1 & 3 & 4 & 2
\end{array}\right),\left(\begin{array}{llll}
1 & 2 & 3 & 4 \\
1 & 3 & 2 & 4
\end{array}\right),\left(\begin{array}{llll}
1 & 2 & 3 & 4 \\
2 & 3 & 1 & 4
\end{array}\right),\left(\begin{array}{llll}
1 & 2 & 3 & 4 \\
2 & 4 & 3 & 1
\end{array}\right) \\
& \left(\begin{array}{llll}
1 & 2 & 3 & 4 \\
2 & 4 & 1 & 3
\end{array}\right),\left(\begin{array}{llll}
1 & 2 & 3 & 4 \\
2 & 1 & 4 & 3
\end{array}\right),\left(\begin{array}{llll}
1 & 2 & 3 & 4 \\
2 & 1 & 3 & 4
\end{array}\right),\left(\begin{array}{llll}
1 & 2 & 3 & 4 \\
2 & 3 & 4 & 1
\end{array}\right) \\
& \left(\begin{array}{llll}
1 & 2 & 3 & 4 \\
3 & 4 & 2 & 1
\end{array}\right),\left(\begin{array}{llll}
1 & 2 & 3 & 4 \\
3 & 2 & 1 & 4
\end{array}\right),\left(\begin{array}{llll}
1 & 2 & 3 & 4 \\
3 & 2 & 4 & 1
\end{array}\right),\left(\begin{array}{llll}
1 & 2 & 3 & 4 \\
3 & 4 & 1 & 2
\end{array}\right) \\
& \left(\begin{array}{llll}
1 & 2 & 3 & 4 \\
3 & 1 & 4 & 2
\end{array}\right),\left(\begin{array}{llll}
1 & 2 & 3 & 4 \\
3 & 1 & 2 & 4
\end{array}\right),\left(\begin{array}{llll}
1 & 2 & 3 & 4 \\
4 & 3 & 1 & 2
\end{array}\right),\left(\begin{array}{llll}
1 & 2 & 3 & 4 \\
4 & 3 & 2 & 1
\end{array}\right) \\
& \left(\begin{array}{llll}
1 & 2 & 3 & 4 \\
4 & 2 & 3 & 1
\end{array}\right),\left(\begin{array}{llll}
1 & 2 & 3 & 4 \\
4 & 2 & 1 & 3
\end{array}\right),\left(\begin{array}{llll}
1 & 2 & 3 & 4 \\
4 & 1 & 2 & 3
\end{array}\right),\left(\begin{array}{llll}
1 & 2 & 3 & 4 \\
4 & 1 & 3 & 2
\end{array}\right) .
\end{aligned}
$$

Apresentaremos a seguir uma forma de simplificar a notação de uma permutação, e a denotamos por notação cíclica.

Definição 3. Sejam $a_{1}, a_{2}, \ldots, a_{r}$ elementos de $X=\{1,2, \ldots, n\}, n>1$, e $\sigma \in \mathrm{S}_{\mathrm{n}}$ uma permutação tal que

$$
\left\{\begin{array}{c}
\sigma\left(\mathrm{a}_{1}\right)=\mathrm{a}_{2} \\
\sigma^{\mathrm{j}}\left(\mathrm{a}_{1}\right)=\mathrm{a}_{\mathrm{j}+1} \\
\sigma^{\mathrm{r}}\left(\mathrm{a}_{1}\right)=\mathrm{a}_{1} \\
\sigma(\mathrm{x})=\mathrm{x} \quad 8 \mathrm{x} \neq \mathrm{a}_{\mathrm{i}}, \mathrm{i}=1, \ldots, \mathrm{r} .
\end{array}\right.
$$

A permutação $\sigma$ é chamada ciclo de comprimento r ou um r-ciclo. O conjunto $\left\{\mathrm{a}_{1}, \ldots, \mathrm{a}_{\mathrm{r}}\right\}$ é chamado suporte do r-ciclo. 
Exemplo 5. Considere a permutação $\sigma=\left(\begin{array}{lllll}1 & 2 & 3 & 4 & 5 \\ 3 & 5 & 4 & 1 & 2\end{array}\right)$ de $\mathrm{S}_{5}$. Vamos representá-la em forma de ciclos. Usando a Definição 3 temos que: $\sigma(1)=3, \sigma^{2}(1)=\sigma(\sigma(1))=\sigma(3)=4, \sigma^{3}(1)=$ $\sigma\left(\sigma^{2}(1)\right)=\sigma(4)=1$, como começamos no 1 e fechamos no 1 , podemos montar o 3-ciclo (134), o próximo passo é verificar para os demais elementos, logo, $\sigma(2)=5, \sigma^{2}(2)=\sigma(\sigma(2))=\sigma(5)=2$; dessa maneira, formamos mais um ciclo, o 2- ciclo (25). Segue assim que nossa permutação pode ser representada pelo produto dos ciclos (134)(25).

Exemplo 6. Agora, vamos representar uma permutação na forma de ciclos usando a noção geométrica. Para isso, considere a permutação $\beta=\left(\begin{array}{cccccccc}1 & 2 & 3 & 4 & 5 & 6 & 7 & 8 \\ 3 & 1 & 6 & 7 & 5 & 2 & 8 & 4\end{array}\right)$ de $\mathrm{S}_{8}$. A figura abaixo mostra o comportamente dessa permutação:

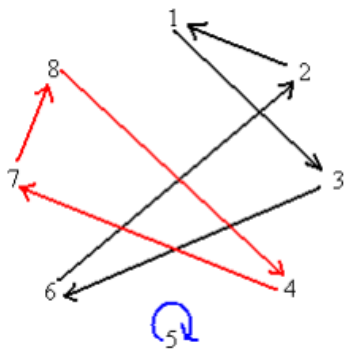

Figura 2: Representação geométrica da permutação

Começando pelo 1 e seguindo a orientação das flechas, formamos o ciclo (1362); dando início pelo 4 forma-se o ciclo (478); e, por fim, compõe-se o ciclo unitário (5). Assim, podemos representar nossa permutação ciclicamente por (1362)(478)(5).

Definição 4. Sejam $\alpha$ um r-ciclo e $\beta$ um s-ciclo de $\mathrm{S}_{\mathrm{n}}$. Os ciclos $\alpha$ e $\beta$ são disjuntos se nenhum elemento de ambos é movido ao mesmo tempo, ou seja, para todo $\mathrm{x} \in\{1,2, \ldots, \mathrm{n}\} \alpha(\mathrm{x})=\mathrm{x}$ ou $\beta(\mathrm{x})=\mathrm{x}$.

Observação 4. Sejam $\alpha$ um r-ciclo e $\beta$ um s-ciclo de $\mathrm{S}_{\mathrm{n}}$. Pensando nos ciclos como conjuntos, dizemos que eles são disjuntos se não têm elementos em comum.

Teorema 1. Dois ciclos disjuntos de uma permutação $\sigma \in \mathrm{S}_{\mathrm{n}}$ comutam entre si.

Demonstração. Considerando $\alpha$ e $\beta$ ciclos disjuntos de $\mathrm{S}_{\mathrm{n}}$, cujos conjuntos suportes são respectivamente $\mathrm{A}$ e $\mathrm{B}$. Se $\mathrm{x} \in \mathrm{I}_{\mathrm{n}}$, temos três casos a analisar:

$1 . \mathrm{x} \in \mathrm{A}$ e $\mathrm{x} \notin \mathrm{B}$. Então, $(\alpha \circ \beta)(\mathrm{x})=\alpha(\beta(\mathrm{x}))=\alpha(\mathrm{x})$, bem como $(\beta \circ \alpha)(\mathrm{x})=\beta(\alpha(\mathrm{x}))=\alpha(\mathrm{x})$. Logo, $\alpha \beta$ e $\beta \alpha$ coincidem em $\mathrm{A}$.

2. $\mathrm{x} \notin \mathrm{A}$ e $\mathrm{x} \in \mathrm{B}$ (Análogo ao 1)

3. $\mathrm{x} \notin \mathrm{A}$ e $\mathrm{x} \notin \mathrm{B}$. Neste caso, $(\alpha \circ \beta)(\mathrm{x})=\alpha(\beta(\mathrm{x}))=\alpha(\mathrm{x})=\mathrm{x}$, assim como, $(\beta \circ \alpha)(\mathrm{x})=\beta(\alpha(\mathrm{x}))$ $=\beta(\mathrm{x})=\mathrm{x}$. Dessa forma, $\alpha \beta$ e $\beta \alpha$ coincidem fora de A e B. 
Exemplo 7. Considere a notação cíclica do Exemplo 6; podemos representá-la por (1362)(478) (5), (5)(478)(1362) ou por (478)(1362)(5); o teorema acima nos diz que é possível comutar os ciclos sem alterar a permutação correspondente.

Teorema 2. Toda permutação de $\sigma \in \mathrm{S}_{\mathrm{n}}$ decompõe-se de forma única, a menos da ordem, em produto ciclos disjuntos.

Exemplo 8. Vamos denotar por $\mathrm{A}_{4, \mathrm{k}}$ o conjunto das permutações de $\mathrm{S}_{4}$ que se decompõem em $\mathrm{k}$ ciclos disjuntos. Representaremos todas as permutações de $\mathrm{S}_{4}$ em produto de ciclos disjuntos, como mostra a tabela abaixo.

\begin{tabular}{|c|c|}
\hline $\mathrm{k}$ & $\mathrm{A}_{4, \mathrm{k}}$ \\
\hline \hline 1 & $(1234),(1324),(1342),(1432),(1243),(1423)$ \\
\hline 2 & $(1)(234),(1)(243),(2)(134),(2)(143),(3)(124),(3)(142),(4)(123),(4)(132),(12)(34),(13)(24),(14)(23)$ \\
\hline 3 & $(1)(2)(34),(1)(3)(24),(1)(4)(23),(2)(3)(14),(2)(4)(13),(3)(4)(12)$ \\
\hline 4 & $(1)(2)(3)(4)$ \\
\hline
\end{tabular}

Tabela 1: Decomposição dos elementos de $\mathrm{S}_{4}$ em produtos de ciclos disjuntos

\section{Os Números de Stirling do Primeiro Tipo}

Os números de Stirling são classificados em dois tipos - números de Stirling do primeiro tipo e segundo tipo - ambos fornecem resultados utilizados para resolver problemas de natureza combinatória. Os números de Stirling do $1^{\mathrm{O}}$ tipo representam o total das permutações que podem ser decompostos um conjunto com $\mathrm{n}$ elementos em $\mathrm{k}$ ciclos disjuntos. Já os do $2^{\mathrm{O}}$ tipo estão relacionados com partições de um conjunto com n elementos.

Os números de Stirling do primeiro tipo podem ser interpretados como o número de maneiras que $\mathrm{n}$ pessoas podem sentar-se em torno de mesas circulares sem que nenhuma dessas mesas fique vazia. Entretanto, exploraremos a natureza combinatória que estabelece uma conexão entre os números de Stirling do primeiro tipo e os grupos de permutação de n elementos. A nomenclatura dada a essa sequência deve-se a uma homenagem imposta pelo matemático dinamarquês Neils Nielsen(18651931) ao matemático escocês James Stirling (1692-1770). Essa homenagem refere-se ao fato de que Stirling utilizava esses números em seus trabalhos, dentre eles, estão os publicados em 1718, intitulado Methodus Differentialis Newtoniana Illustrata e em seu livro Methodus Differentiallis. Nesta seção apresentamos alguns dos resultados sobre os números de Stirling que decorrem de [2], sendo um desses uma fórmula de recorrência que descreve os números de Stirling do primeiro tipo. Além disso, descrevemos algumas propriedades inerentes desse conceito.

Definição 5. Sejam $n, k \in \mathbb{N}$, com $n \geq k \geq 1$, o número de Stirling de primeiro tipo é o total de permutações de $\mathrm{n}$ elementos que se decompõem em exatamente $\mathrm{k}$ ciclos disjuntos. Denotamos o número de Stirling de primeiro tipo por $\left[\begin{array}{l}\mathrm{n} \\ \mathrm{k}\end{array}\right]$. Convencionamos $\left[\begin{array}{l}0 \\ 0\end{array}\right]=1$.

Segue diretamente da definição 5 que: $\left[\begin{array}{l}\mathrm{n} \\ 0\end{array}\right]=0$, para $\mathrm{n}>0 ;\left[\begin{array}{l}\mathrm{n} \\ \mathrm{n}\end{array}\right]=1 ;\left[\begin{array}{l}\mathrm{n} \\ \mathrm{k}\end{array}\right]=0$, para $0<\mathrm{n}<\mathrm{k}$.

Exemplo 9. Vamos calcular o número de Stirling $\left[\begin{array}{l}4 \\ 2\end{array}\right]$; para isso, basta olhar quantas permutações de 4 elementos decompõem-se em 2 ciclos disjuntos e, pela tabela do Exemplo 8, temos que 
a resposta é 11 . Ou seja, $\left[\begin{array}{l}4 \\ 2\end{array}\right]=11$. Vale observar que o método utilizado para calcular os números de Stirling para qualquer $\mathrm{n} \in \mathbb{N}$ segue de modo análogo ao que fizemos aqui, mas, quanto maior for $\mathrm{n}$ mais difícil fica calcular esses números manualmente; assim, recorrer aos programas computacionais é a melhor escolha. A título de curiosidade, se quiséssemos calcular os números de Stirling para $\mathrm{n}=5$, deveríamos primeiro calcular todas as permutações de $\mathrm{S}_{5}$ e depois decompô-las em ciclos disjuntos, mas, sabemos que a ordem de $S_{5}$ é 5 ! = 120, então, para $n \geq 4$ torna-se mais trabalhoso calcular os números de Stirling manualmente. A seguir dispomos uma relação que nos permite facilitar o cálculo desses números.

Teorema 3. Sejam $\mathrm{n}, \mathrm{k}$ números naturais com $\mathrm{n} \geq \mathrm{k} \geq 1$, temos os seguintes resultados:

a) $\left[\begin{array}{c}n \\ 1\end{array}\right]=(\mathrm{n}-1) !$

b) $\left[\begin{array}{c}\mathrm{n} \\ \mathrm{n}-1\end{array}\right]=\left(\begin{array}{l}\mathrm{n} \\ 2\end{array}\right)$.

Demonstração. a) Temos que $\left[\begin{array}{c}\mathrm{n} \\ 1\end{array}\right]$ representa o total de permutações $\sigma \in \mathrm{S}_{\mathrm{n}}$ com exatamente 1 ciclo, assim, podemos formar $(n-1)$ ! permutações com exatamente 1 ciclo a partir de $1,2, \ldots, n$, ou seja,

$\begin{array}{ccccc}(1 & 2 & 3 & \ldots & \mathrm{n}) \\ (2 & 1 & 3 & \ldots & \mathrm{n}) \\ (3 & 1 & 4 & \ldots & \mathrm{n}) \\ \vdots & \vdots & \vdots & \ddots & \vdots \\ (\mathrm{n}-1 & \mathrm{n}-2 & \mathrm{n}-3 & \ldots & \mathrm{n}) .\end{array}$

b) Temos que $\left[\begin{array}{c}n \\ n-1\end{array}\right]$ é o total de permutações $\sigma \in \mathrm{S}_{\mathrm{n}}$ decomposta em um produto de $\mathrm{n}-1$ ciclos. Para formar permutações de $n-1$ ciclos, temos que escolher dois elementos de 1,2, .., $\mathrm{n}$ para ser uma transposição e o restante fica pré-fixado para ser o ciclo de comprimento 1. Dessa forma, $\left[\begin{array}{c}\mathrm{n} \\ \mathrm{n}-1\end{array}\right]=\left(\begin{array}{c}\mathrm{n} \\ 2\end{array}\right)$.

Definição 6. Os coeficientes binomiais são números inteiros positivos definidos por:

$$
\left(\begin{array}{l}
\mathrm{n} \\
\mathrm{k}
\end{array}\right)=\frac{\mathrm{n} !}{\mathrm{k} !(\mathrm{n}-\mathrm{k}) !},
$$

com $\mathrm{n}, \mathrm{k} \in \mathbb{N}, \mathrm{n} \geq \mathrm{k}$.

Esses coeficientes podem ser interpretados como o número de maneiras de combinar n elementos tomados k a k. Além disso, os números binomiais satisfazem uma relação de recorrência na qual o nome foi dado em homenagem ao célebre matemático Michael Stifel (1487-1567), a relação de Stifel ou também conhecida como regra de Pascal, cuja identidade envolve os coeficientes binomiais. Essa relação é dada por:

$$
\left(\begin{array}{l}
\mathrm{n} \\
\mathrm{k}
\end{array}\right)=\left(\begin{array}{c}
\mathrm{n}-1 \\
\mathrm{k}-1
\end{array}\right)+\left(\begin{array}{c}
\mathrm{n}-1 \\
\mathrm{k}
\end{array}\right),
$$


com $\mathrm{n} \geq \mathrm{k}$ naturais.

Por exemplo, seja $A=\{1,2,3,4\}$, em quantas maneiras podemos organizar esses números tomados 2 a 2 ? Como temos uma quantidade finita e pequena de elementos, o trabalho manual é bem simples, mas, recorrendo à definição dada acima, temos que o número de possibilidades é dado por

$$
\left(\begin{array}{l}
4 \\
2
\end{array}\right)=\frac{4 !}{2 !(4-2) !}=\frac{4 !}{\cdot 2 !(2 !)}=\frac{4.3 \cdot 2 !}{2 ! 2 !}=\frac{4.3}{2}=6
$$

ou seja, temos 6 maneiras de agrupar esses 4 elementos tomados de 2 em 2 .

Exemplo 10. Considere o grupo simétrico $\mathrm{S}_{4}$, vamos calcular $\left[\begin{array}{l}4 \\ 1\end{array}\right]$ e $\left[\begin{array}{l}4 \\ 3\end{array}\right]$. Pelo teorema 3 é fácil calcular esses números. Sendo assim: $\left[\begin{array}{l}4 \\ 1\end{array}\right]=(4-1) !=3 !=6$ e $\left[\begin{array}{l}4 \\ 3\end{array}\right]=\left(\begin{array}{l}4 \\ 2\end{array}\right)=\frac{4 !}{2 !(4-2) ! \bullet}=6$.

O teorema a seguir fornece-nos uma recorrência para os números de Stirling do primeiro tipo que se assemelha à relação de Stifel.

Teorema 4. Sejam $\mathrm{n}, \mathrm{k} \in \mathbb{N}$ com $\mathrm{n} \geq \mathrm{k} \geq 1$. então:

$$
\left[\begin{array}{l}
\mathrm{n} \\
\mathrm{k}
\end{array}\right]=\left[\begin{array}{l}
\mathrm{n}-1 \\
\mathrm{k}-1
\end{array}\right]+(\mathrm{n}-1)\left[\begin{array}{c}
\mathrm{n}-1 \\
\mathrm{k}
\end{array}\right]
$$

Demonstração. Inicialmente vamos indicar por A o conjunto de permutações de $\mathrm{S}_{\mathrm{n}}$ que se decompõem em exatamente $\mathrm{k}$ ciclos. Segue da definição que $|\mathrm{A}|=\left[\begin{array}{l}\mathrm{n} \\ \mathrm{k}\end{array}\right]$. Particionamos $\mathrm{A}$ em dois subconjuntos:

1 . Subconjunto de $\mathrm{A}, \mathrm{A}_{1}$, formado por permutações que contém o 1-ciclo (n);

2 . Subconjunto de $\mathrm{A}, \mathrm{A}_{2}$, formado por permutações cujo elemento n está em um ciclo com mais de um elemento.

Temos que: $A_{1} \cap A_{2}=\varnothing$. e $A_{1} \cup A_{2}=A$. Em 1, o total de permutações é $\left[\begin{array}{c}n-1 \\ k-1\end{array}\right]=\left|A_{1}\right|$, em 2 o total de permutações é $(n-1)\left[\begin{array}{c}n-1 \\ k\end{array}\right]=\left|A_{2}\right|$, pois podemos agora distribuir todos os $\mathrm{n}-1$ elementos em $\mathrm{k}$ ciclos - afinal, cada ciclo pode ter mais de um elemento - e assim, obtemos $\left[\begin{array}{c}\mathrm{n}-1 \\ \mathrm{k}\end{array}\right]$ modos. Como temos $\mathrm{n}-1$ modos de colocar o elemento n, sem contar sua própria posição, alcançamos pelo princípio multiplicativo $(n-1)\left[\begin{array}{c}n-1 \\ k\end{array}\right]$ permutações de $S_{n}$ em $A_{2}$. Logo,

$$
\left[\begin{array}{l}
\mathrm{n}-1 \\
\mathrm{k}-1
\end{array}\right]+(\mathrm{n}-1)\left[\begin{array}{c}
\mathrm{n}-1 \\
\mathrm{k}
\end{array}\right]=|\mathrm{A}|=\left[\begin{array}{l}
\mathrm{n} \\
\mathrm{k}
\end{array}\right] \text {. }
$$


Exemplo 11. Seja $\mathrm{S}_{4}$ o grupo simétrico de grau 4. Calculamos $\left[\begin{array}{l}5 \\ 2\end{array}\right]$ por meio do teorema anterior. Sendo assim:

$$
\left[\begin{array}{l}
5 \\
3
\end{array}\right]=\left[\begin{array}{l}
5-1 \\
3-1
\end{array}\right]+(5-1)\left[\begin{array}{c}
5-1 \\
3
\end{array}\right]=\left[\begin{array}{l}
4 \\
2
\end{array}\right]+(4)\left[\begin{array}{l}
4 \\
3
\end{array}\right]=11+4.6=35,
$$

o resultado da terceira igualdade foi obtido por meio da Tabela 8.

Exemplo 12. Pelo teorema anterior vimos que a recorrência para os números de Stirling assemelhase à relação de Stifel, que nos fornece o triângulo de Pascal. Para os números de Stirling do primeiro tipo temos um triângulo análogo, como mostra a figura abaixo:

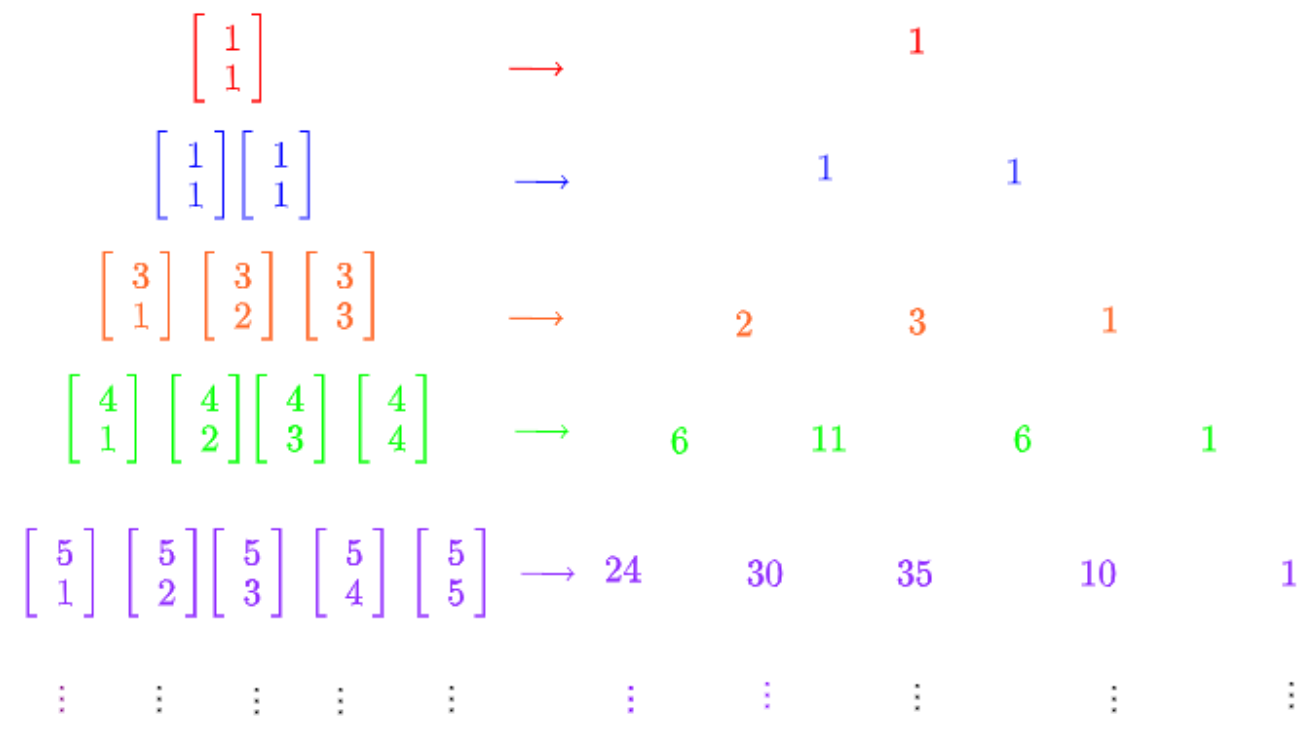

Figura 3: Triângulo similar ao de Pascal para os números de Stirling do primeiro tipo

Teorema 5. Sejam $\mathrm{n}, \mathrm{k} \in \mathbb{N}$ com $\mathrm{n} \geq \mathrm{k} \geq 1$. então:

$$
\sum_{\mathrm{k}=0}^{\mathrm{n}}\left[\begin{array}{l}
\mathrm{n} \\
\mathrm{k}
\end{array}\right]=\mathrm{n} !
$$

Demonstração. Faremos a prova por indução sobre n. Para $\mathrm{n}=0$, temos que:

$$
\sum_{\mathrm{k}=0}^{\mathrm{n}}\left[\begin{array}{l}
0 \\
\mathrm{k}
\end{array}\right]=\left[\begin{array}{l}
0 \\
0
\end{array}\right]=1=0 !
$$


Suponha que o resultado seja válido para $\mathrm{n}=\mathrm{t}>0$, ou seja, $\sum_{\mathrm{k}=0}^{\mathrm{t}}\left[\begin{array}{c}\mathrm{t} \\ \mathrm{k}\end{array}\right]=\mathrm{t}$ !. Assim, temos que:

$$
\begin{aligned}
\sum_{\mathrm{k}=0}^{\mathrm{t}+1}\left[\begin{array}{c}
\mathrm{t}+1 \\
\mathrm{k}
\end{array}\right] & =\sum_{\mathrm{k}=0}^{\mathrm{t}+1}\left(\left[\begin{array}{c}
\mathrm{t} \\
\mathrm{k}-1
\end{array}\right]+\mathrm{t}\left[\begin{array}{l}
\mathrm{t} \\
\mathrm{k}
\end{array}\right]\right)=\sum_{\mathrm{k}=0}^{\mathrm{t}+1}\left[\begin{array}{c}
\mathrm{t} \\
\mathrm{k}-1
\end{array}\right]+\mathrm{t} \sum_{\mathrm{k}=0}^{\mathrm{t}+1}\left[\begin{array}{c}
\mathrm{t} \\
\mathrm{k}
\end{array}\right] \\
& =\sum_{\mathrm{k}=0}^{\mathrm{t}+1}\left[\begin{array}{c}
\mathrm{t} \\
\mathrm{k}-1
\end{array}\right]+\mathrm{t} \sum_{\mathrm{k}=0}^{\mathrm{t}}\left[\begin{array}{c}
\mathrm{t} \\
\mathrm{k}
\end{array}\right]+\mathrm{t}\left[\begin{array}{c}
\mathrm{t} \\
\mathrm{t}+1
\end{array}\right] \\
& =\sum_{\mathrm{k}=0}^{\mathrm{t}+1}\left[\begin{array}{c}
\mathrm{t} \\
\mathrm{k}-1
\end{array}\right]+\mathrm{tt} !=\sum_{\mathrm{p}=\mathrm{o}}^{\mathrm{t}}\left[\begin{array}{l}
\mathrm{t} \\
\mathrm{p}
\end{array}\right]+\mathrm{tt} !=\mathrm{t} !+\mathrm{tt} !=(\mathrm{t}+1) !
\end{aligned}
$$

Portanto, $\sum_{\mathrm{k}=0}^{\mathrm{n}}\left[\begin{array}{l}\mathrm{n} \\ \mathrm{k}\end{array}\right]=\mathrm{n}$ !.

Exemplo 13. Perceba pela Figura 3 que a soma de cada linha do triângulo corresponde a n!, por exemplo, a soma dos elementos da quarta linha é

$$
\sum_{\mathrm{k}=1}^{4}\left[\begin{array}{l}
4 \\
\mathrm{k}
\end{array}\right]=6+11+6+1=24=4 !
$$

note que o resultado segue diretamente do Teorema 5.

A relação de recorrência estabelecida no Teorema 4 permite determinar a função geradora ordinária para essa sequência de números, ou seja, podemos obter um polinômio de grau n, cujo coeficiente de $\mathrm{x}^{\mathrm{k}}$ é o número de Striling do primeiro tipo. De fato, considere

$$
\mathrm{F}_{\mathrm{n}}(\mathrm{x})=\sum_{\mathrm{k}=1}^{\infty}\left[\begin{array}{l}
\mathrm{n} \\
\mathrm{k}
\end{array}\right] \mathrm{x}^{\mathrm{k}}
$$

com $n \geq k \geq 1$, em que $n$ é um inteiro fixado, convencionamos $F_{0}(x)=1$. Assim temos que

$$
\left[\begin{array}{l}
n \\
k
\end{array}\right] x^{n}=\left[\begin{array}{l}
n-1 \\
k-1
\end{array}\right] x^{n}+(n-1)\left[\begin{array}{c}
n-1 \\
k
\end{array}\right] x^{n}
$$

Dessa forma,

$$
\sum_{\mathrm{k}=1}^{\infty}\left[\begin{array}{l}
\mathrm{n} \\
\mathrm{k}
\end{array}\right] \mathrm{x}^{\mathrm{k}}=\sum_{\mathrm{k}=1}^{\infty}\left[\begin{array}{l}
\mathrm{n}-1 \\
\mathrm{k}-1
\end{array}\right] \mathrm{x}^{\mathrm{k}}+\sum_{\mathrm{k}-1}^{\infty}(\mathrm{n}-1)\left[\begin{array}{c}
\mathrm{n}-1 \\
\mathrm{k}
\end{array}\right] \mathrm{x}^{\mathrm{k}}, \mathrm{k} \geq 1
$$

Portanto:

$$
\mathrm{F}_{\mathrm{n}}(\mathrm{x})=\mathrm{x} \sum_{\mathrm{k}=1}^{\infty}\left[\begin{array}{c}
\mathrm{n}-1 \\
\mathrm{k}-1
\end{array}\right] \mathrm{x}^{\mathrm{k}-1}+(\mathrm{n}-1) \sum_{\mathrm{k}=1}^{\infty}\left[\begin{array}{c}
\mathrm{n}-1 \\
\mathrm{k}
\end{array}\right] \mathrm{x}^{\mathrm{k}}
$$

ou seja, $F_{n}(x)=x F_{n-1}(x)+(n-1) F_{n-1}(x)$, ou ainda, $F_{n}(x)=(x+n-1) F_{n-1}(x), 8 n \geq 1$. Avaliamos a função $F_{n}(x)$ para $n=1,2,3, \ldots$

$$
\begin{aligned}
\mathrm{F}_{1}(\mathrm{x}) & =(\mathrm{x}+1-1) \cdot \mathrm{F}_{1-1}(\mathrm{x})=\mathrm{x} \cdot \mathrm{F}_{0}(\mathrm{x})=\mathrm{x} \\
\mathrm{F}_{2}(\mathrm{x}) & =(\mathrm{x}+2-1) \cdot \mathrm{F}_{2-1}(\mathrm{x})=(\mathrm{x}+1) \cdot \mathrm{x} \\
& \vdots \\
\mathrm{F}_{\mathrm{n}}(\mathrm{x}) & =(\mathrm{x}+\mathrm{n}-1) \ldots(\mathrm{x}+4)(\mathrm{x}+3)(\mathrm{x}+2)(\mathrm{x}+1) \mathrm{x} .
\end{aligned}
$$

Dessa forma, ao expandirmos o polinômio $\mathrm{F}_{\mathrm{n}}(\mathrm{x})$, o coeficiente de $\mathrm{x}^{\mathrm{k}}$ é o número de Stirling do primeiro tipo. 
Teorema 6. Seja o inteiro n tal que $\mathrm{n}>0$, então a função geradora ordinária para os números de Stirling do primeiro tipo é o polinômio na indeterminada $\mathrm{x}$

$$
\mathrm{F}_{\mathrm{n}}(\mathrm{x})=(\mathrm{x}+\mathrm{n}-1) \ldots(\mathrm{x}+4)(\mathrm{x}+3)(\mathrm{x}+1) \mathrm{x} .
$$

Exemplo 14. Considere a seguinte identidade em particular:

$$
\sum_{k=1}^{4} k\left[\begin{array}{l}
4 \\
k
\end{array}\right]=\left[\begin{array}{c}
4+1 \\
2
\end{array}\right]=\left[\begin{array}{l}
5 \\
2
\end{array}\right]
$$

Temos que $\left[\begin{array}{l}5 \\ 2\end{array}\right]=50$.

No caso $\mathrm{k}=1$, lado esquerdo da identidade (1), considerando as permutações de $\{1,2,3,4\}$ que se decompõem em um ciclo, e transformando-as em permutações de $\{1,2,3,4,5\}$ que se decompõem em produtos de dois ciclos, temos que:

$$
\begin{aligned}
& (2341) \rightarrow(2341)(5) \\
& (2413) \rightarrow(2413)(5) \\
& (3421) \rightarrow(3421)(5) \\
& (1342) \rightarrow(1342)(5) \\
& (4312) \rightarrow(4312)(5) \\
& (4123) \rightarrow(4123)(5)
\end{aligned}
$$

Temos um total de 6 permutações de $\{1,2,3,4,5\}$, que se decompõem em produto de dois ciclos. No caso $k=2$, para cada permutação de $\{1,2,3,4\}$ que se separam em dois ciclos, vamos repeti-las duas vezes, sublinhando um dos ciclos para diferenciá-las, como segue:
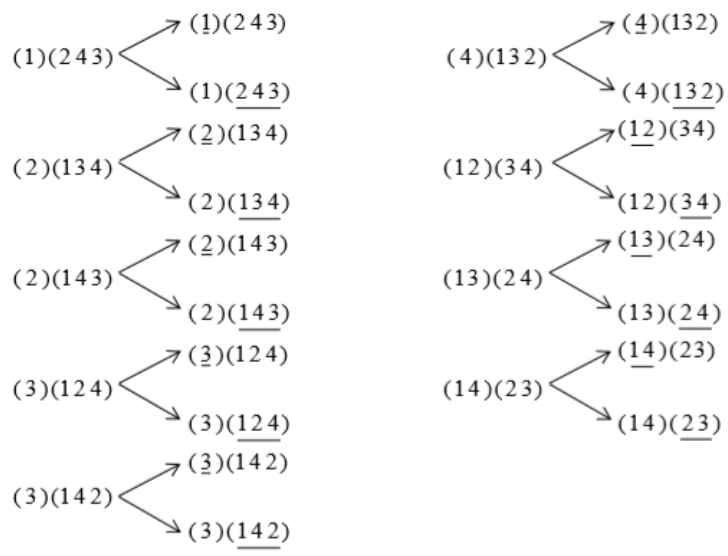

Figura 4: Caso $\mathrm{k}=2$

Para cada uma das permutações duplicadas, vamos associá-la às seguintes permutações de $\{1,2,3,4,5\}$ que se decompõem em produto de dois ciclos. 

$(1)(234) \rightarrow(1)(2345) ;$
(1)(234) $\rightarrow$ (15)(234);
$(1)(243) \rightarrow(1)(2435)$;
(1)(243) $\rightarrow$ (15)(243);
$(\underline{2})(134) \rightarrow(2)(1345) ;$
$(2)(134) \rightarrow(25)(134)$;
$(2)(143) \rightarrow(2)(1435)$;
$(2)(143) \rightarrow(25)(143)$;
$(\underline{3})(124) \rightarrow(3)(1245)$;
(3)(124) $\rightarrow$ (35)(124);
$(\underline{3})(142) \rightarrow(3)(1425)$;
$(3)(142) \rightarrow(35)(142)$;

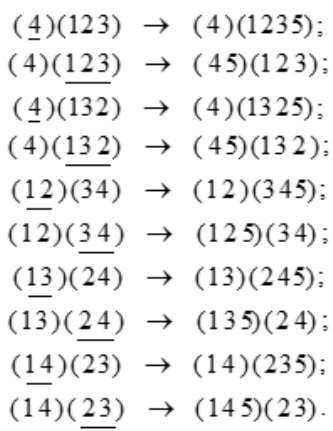

Figura 5: Permutações de 4 associadas a permutações de 5.

Temos um total de 22 permutações de $\{1,2,3,4,5\}$, que se decompõem em produto de dois ciclos. No caso $\mathrm{k}=3$, para cada permutação de $\{1,2,3,4\}$ que se decompõe em três ciclos, a repetimos três vezes, sublinhando um dos ciclos para diferenciá-los, como segue:

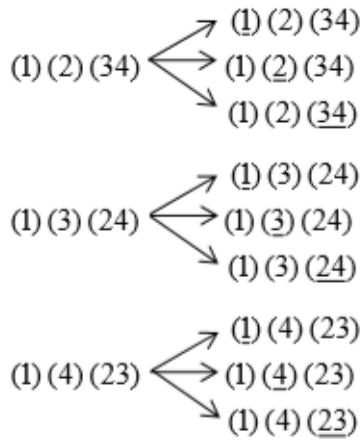

(2) (3) (14) $\longleftrightarrow_{(2)(3)(14)}$

(2) $(4)$

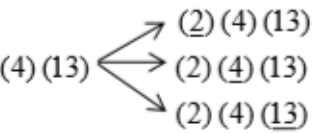

(3) (4) (12) (3) (4) (12)

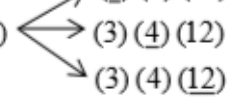

Figura 6: Caso $\mathrm{k}=3$.

Para cada uma das permutações triplicadas, associamos a seguinte permutação de $\{1,2,3,4,5\}$ que se decompõem em produto de dois ciclos.

Temos um total de 18 permutações de $\{1,2,3,4,5\}$, que se decompõem em produto de dois ciclos. No caso $\mathrm{k}=4$, para cada permutação de $\{1,2,3,4\}$ que se decompõem em quatro ciclos, a saber, (1)(2)(3)(4), as repetimos quatro vezes, sublinhando um dos ciclos para diferenciá-los.

Para cada uma das permutações quadruplicadas, que diferenciamos sublinhando um dos ciclos, associamos uma permutação de $\{1,2,3,4,5\}$ que se decompõem em produto de dois ciclos.

$$
\begin{aligned}
& (\underline{1})(2)(3)(4) \rightarrow \text { (5432)(1) } \\
& (1)(\underline{2})(3)(4) \rightarrow \quad(5431)(2) \\
& (1)(2)(\underline{3})(4) \rightarrow \text { (5241)(3) } \\
& (1)(2)(3)(\underline{4}) \rightarrow \text { (5321)(4) }
\end{aligned}
$$



(1) (2) (34) $\rightarrow$ (1)(5342)
(1) (4) (23) $\rightarrow$ (1)(5234)
(2) (4) (13) $\rightarrow$ (2) (5134)
(1) (2) (34) $\rightarrow$ (2) (5134)
(1) (4) (23) $\rightarrow$ (4) (5 231)
(2) (4) (13) $\rightarrow$ (4)(5132)
(1) (2) (34) $\rightarrow$ (34) (521)
(1) $(4)(\underline{23}) \rightarrow(23)(541)$
$(2)(4)(\underline{13}) \rightarrow(13)(542)$
(1) $(3)(24) \rightarrow(1)(5243)$
(2) (3) (14) $\rightarrow$ (2) (5143)
(3) (4) (12) $\rightarrow$ (3) (5124)
(1) (3) $(24) \rightarrow(3)(5241)$
$(2)(\underline{3})(14) \rightarrow(3)(5142)$
(3) (4) (12) $\rightarrow$ (4)(5123)
(1) (3) (24) $\rightarrow$ (24) (531)
(2) (3) $(\underline{14}) \rightarrow(14)(532)$
$(3)(4)(\underline{12}) \rightarrow(12)(543)$

Figura 7: Permutações de 4 que se decompõem em 3 ciclos associadas a permutações de 5.

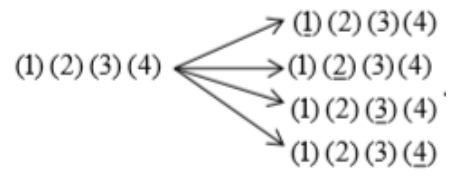

Figura 8: Caso $\mathrm{k}=4$.

Observe que obtemos um total de 50 permutações de $\{1,2,3,4,5\}$ que se decompõem em produto de dois ciclos.

Proposição 1. Sejam $\mathrm{n}$ e $\mathrm{k}$ números naturais, com $\mathrm{n}, \mathrm{k} \geq 0$, temos que

$$
\sum_{\mathrm{k}=1}^{\mathrm{n}} \mathrm{k}\left[\begin{array}{l}
\mathrm{n} \\
\mathrm{k}
\end{array}\right]=\left[\begin{array}{c}
\mathrm{n}+1 \\
2
\end{array}\right]
$$

A demonstração dessa proposição segue de modo análogo ao desenvolvimento do exemplo 2.

\section{Números Harmônicos que são racionais}

Esta seção foi fundamentada por meio do artigo [4] em que diversas propriedades envolvem números harmônicos e números de Stirling. Os números harmônicos são somas parciais da série harmônica. O estudo e entendimento da série harmônica [1] teve origem no século VI antes de Cristo com as experiências feitas pelo filósofo e matemático grego Pitágoras. Pelas suas descobertas é possível estabelecer uma relação direta entre melodia e harmonia, sendo que seus conceitos e definições são utilizados até os dias atuais (oitavas, ciclo de quintas etc).

Pitágoras (séc. VI, a.C.), afirmou que qualquer som para ser musical teria que ter altura definida e ser emitido por um instrumento ou por fonte natural, resultando em uma vibração ondulatória regular. Essa vibração é composta pelo som gerador (1 $1^{a}$ nota) e outros sons definidos de intensidade menor e frequência mais aguda, chamados de sons harmônicos ou série harmônica.

A série harmônica (som gerador + notas agudas subsequentes) apresenta uma relação intervalar característica e imutável de origem natural ou física. Se tomarmos como exemplo uma corda 
de um violão ( $6^{\mathrm{a}}$ Corda - Nota Mi Grave) pode-se observar que além de vibrar em toda a sua extensão, também vibra em sua metade, em sua terça parte, em sua quarta parte e quinta parte etc., produzindo sons cada vez mais agudos.

Inicialmente definimos e exemplificamos os números harmônicos e apresentamos algumas propriedades. Por fim, estabelecemos sua relação com os Números de Stirling do primeiro tipo.

Os números Harmônicos são definidos como sendo a soma dos inversos naturais, ou seja,

$$
\mathrm{H}_{\mathrm{n}}=1+\frac{1}{2}+\frac{1}{3}+\cdots+\frac{1}{\mathrm{n}}=\sum_{\mathrm{k}=1}^{\mathrm{n}} \frac{1}{\mathrm{k}}
$$

com $\mathrm{n} \geq 1$. Vamos convencionar $\mathrm{H}_{0}=0$.

As séries harmônicas (ou também conhecidas como séries-p) são da forma $\sum_{\mathrm{k}=1}^{\mathrm{n}} \frac{1}{\mathrm{k}^{\mathrm{p}}}$. Em termos de convergência dizemos que essas séries convergem-se $\mathrm{p}<1$ e divergem se $\mathrm{p} \geq 1$. No nosso caso, $\mathrm{p}=1$, logo, nossa série é divergente, ou seja, a somatória não nos dá um número finito. Mais informações sobre convergência de séries podem ser vistas em [9].

Os números harmônicos aparecem como aplicações em outras ciências; um exemplo é na música, para isso veja [7]. A seguir enunciamos e provamos uma propriedade para os números harmônicos.

Proposição 2. Seja n um número natural tal que $\mathrm{n} \geq 1$, temos que:

$$
\sum_{\mathrm{k}=1}^{\mathrm{n}-1} \mathrm{H}_{\mathrm{k}}=\mathrm{nH}_{\mathrm{n}}-\mathrm{n}
$$

Demonstração. Temos que

$$
\sum_{\mathrm{k}=1}^{\mathrm{n}-1} \mathrm{H}_{\mathrm{k}}=\mathrm{H}_{1}+\mathrm{H}_{2}+\mathrm{H}_{3}+\cdots+\mathrm{H}_{\mathrm{n}-1} \text {. }
$$

ou seja,

$$
\begin{aligned}
\sum_{\mathrm{k}=1}^{\mathrm{n}-1} \mathrm{H}_{\mathrm{k}} & =1+1+\frac{1}{2}+1+\frac{1}{2}+\frac{1}{3}+\cdots+1+\frac{1}{2}+\cdots+\frac{1}{\mathrm{n}-1} \\
& =(\mathrm{n}-1) \cdot 1+(\mathrm{n}-2) \cdot \frac{1}{2}+\cdots+(\mathrm{n}-3) \cdot \frac{1}{3}+\cdots+[(\mathrm{n}-(\mathrm{n}-1))] \cdot \frac{1}{\mathrm{n}-1}
\end{aligned}
$$

Assim temos:

$$
\begin{aligned}
\sum_{\mathrm{k}=1}^{\mathrm{n}-1} \mathrm{H}_{\mathrm{k}} & =\mathrm{n}+\frac{\mathrm{n}}{2}+\frac{\mathrm{n}}{3}+\cdots+\frac{\mathrm{n}}{\mathrm{n}-1} \underbrace{-1-1-1-1}_{\mathrm{n}-1} \\
& =\mathrm{n}+\frac{\mathrm{n}}{2}+\frac{\mathrm{n}}{3}+\cdots+\frac{\mathrm{n}}{\mathrm{n}-1}-\mathrm{n}+1 \\
& =\mathrm{n}+\frac{\mathrm{n}}{2}+\frac{\mathrm{n}}{3}+\cdots+\frac{\mathrm{n}}{\mathrm{n}-1}-\mathrm{n}+\frac{\mathrm{n}}{\mathrm{n}} \\
& =\mathrm{n}\left(1+\frac{1}{2}+\frac{1}{3}+\cdots+\frac{1}{\mathrm{n}-1}+\frac{1}{\mathrm{n}}\right)-\mathrm{n}=\mathrm{nH}_{\mathrm{n}}-\mathrm{n} .
\end{aligned}
$$


Teorema 7. Seja n natural, $\mathrm{n} \geq 1$, temos que:

$$
\mathrm{H}_{\mathrm{n}}=\frac{1}{\mathrm{n} !}\left[\begin{array}{c}
\mathrm{n}+1 \\
2
\end{array}\right]
$$

Demonstração.

$$
\begin{aligned}
\frac{1}{n !}\left[\begin{array}{c}
n+1 \\
2
\end{array}\right] & =\frac{\left[\begin{array}{c}
n+1 \\
2
\end{array}\right]}{n !}=\frac{\left[\begin{array}{l}
n \\
1
\end{array}\right]+n\left[\begin{array}{l}
n \\
2
\end{array}\right]}{n !}=\frac{\left[\begin{array}{l}
n \\
1
\end{array}\right]}{n !}+\frac{n\left[\begin{array}{l}
n \\
2
\end{array}\right]}{n !} \\
& =\frac{(n-1) !}{n !}+\frac{\left[\begin{array}{l}
n \\
2
\end{array}\right]}{(n-1) !}=\frac{1}{n}+\frac{\left[\begin{array}{l}
n \\
2
\end{array}\right]}{(n-1) !}
\end{aligned}
$$

Segue da equação (2) que

$$
\frac{\left[\begin{array}{c}
\mathrm{n} \\
2
\end{array}\right]}{(\mathrm{n}-1) !}=\frac{1}{(\mathrm{n}-1) !}+\frac{\left[\begin{array}{c}
\mathrm{n}-1 \\
2
\end{array}\right]}{(\mathrm{n}-2) !}
$$

Substituindo (3) em (2) temos:

$$
\frac{1}{n !}\left[\begin{array}{c}
n+1 \\
2
\end{array}\right]=\frac{1}{n}+\frac{1}{n-1}+\frac{\left[\begin{array}{c}
n \\
2
\end{array}\right]}{(n-2) !} .
$$

De forma análoga

$$
\frac{\left[\begin{array}{c}
\mathrm{n}-1 \\
2
\end{array}\right]}{(\mathrm{n}-2) !}=\frac{1}{\mathrm{n}-2}+\frac{\left[\begin{array}{c}
\mathrm{n}-2 \\
2
\end{array}\right]}{(\mathrm{n}-3) !} .
$$

Repetindo esse processo temos que:

$$
\frac{1}{n !}\left[\begin{array}{c}
n+1 \\
2
\end{array}\right]=\frac{1}{n}+\frac{1}{n-1}+\cdots+\frac{1}{2}+1=H_{n} .
$$

Portanto,

$$
\mathrm{H}_{\mathrm{n}}=\frac{1}{\mathrm{n} !}\left[\begin{array}{c}
\mathrm{n}+1 \\
2
\end{array}\right]
$$




\section{Uma possível abordagem dos Números de Stirling no ensino médio}

Segundo a Base Nacional Comum Curricular (BNCC) os estudantes do ensino médio devem ter a habilidade de "Resolver e elaborar problemas de contagem envolvendo diferentes tipos de agrupamento de elementos [...]"; nesse viés, vimos anteriormente que os números de Stirling servem como ferramenta para contar em quantos ciclos disjuntos uma permutação decompõe-se, mas, além de seu caráter combinatório, os números de Stirling do primeiro tipo podem ser desenvolvidos por meio de seu caráter algébrico, para isso, consideremos o polinômio definido por

$$
\mathrm{F}_{\mathrm{n}}(\mathrm{x})=(\mathrm{x}+\mathrm{n})(\mathrm{x}+(\mathrm{n}-1)) \ldots(\mathrm{x}+1) \mathrm{x}
$$

para $\mathrm{n}>0$ e por convenção $\mathrm{F}_{0}(\mathrm{x})=1$. O coeficiente de $\mathrm{x}^{\mathrm{k}}$ na expansão de $\mathrm{F}_{\mathrm{n}}(\mathrm{x})$ é $\left[\begin{array}{l}\mathrm{n} \\ \mathrm{k}\end{array}\right]$.

No ensino médio, os números de Stirling podem ser abordados dessa maneira. Além disso, também é possível explorar a natureza combinatória desses números por meio do seguinte problema: qual é o número de maneiras de $\mathrm{n}$ pessoas sentarem-se em $\mathrm{k}$ mesas circulares idênticas sem que nenhuma delas fique vazia? A resposta para o problema é: $\left[\begin{array}{l}\mathrm{n} \\ \mathrm{k}\end{array}\right]$, com $\mathrm{n} \geq \mathrm{k}>0$.

O exemplo a seguir dá-nos ideia da natureza combinatória dos números de Stirling do primeiro tipo.

Exemplo 15. De quantas maneiras quatro pessoas podem sentar-se em volta de três mesas circulares idênticas, sem que nenhuma mesa fique vazia?

Esse problema representa uma contagem, em que o número de mesas representa os ciclos, e o número de pessoas os elementos do conjunto A, ou seja, como temos quatro pessoas, o conjunto A poderá ser definido por $\mathrm{A}=\{\mathrm{a}, \mathrm{b}, \mathrm{c}, \mathrm{d}\}$.

Do enunciado do exemplo sabemos que temos três mesas. Abaixo estão representadas geometricamente as maneiras de organizarmos essa distribuição - vale observar que as letras representam as pessoas e os cículos representam as mesas:

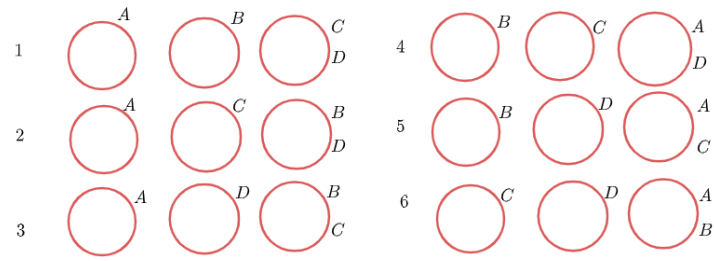

Figura 9: Distribuição de 4 pessoas em torno de 3 mesas circulares idênticas

Neste caso, temos 6 maneiras de realizar a distribuição, ou seja, $\left[\begin{array}{l}4 \\ 3\end{array}\right]$.

Além disso, o item a) do Teorema 3 é um caso particular de permutação circular, pois o número de Stirling do primeiro tipo $\left[\begin{array}{l}\mathrm{n} \\ 1\end{array}\right]$ pode ser interpretado como a distribuição de $\mathrm{n}$ pessoas em torno de 1 mesa circular, e essa é a definição de permutação circular. Dessa forma, podemos interpretar 
a permutação circular como um caso particular dos números de Stirling do primeiro tipo $\left[\begin{array}{l}\mathrm{n} \\ \mathrm{k}\end{array}\right]$, quando $\mathrm{k}=1$ e $\mathrm{n}>0$. Vemos assim que os números de Stirling do primeiro tipo podem ser explorados no ensino médio de forma combinatória por meio das permutações circulares. Em [10] é explorada a relação entre as permutações circulares e os números de Stirling do primeiro tipo.

\section{Referências}

[1] Anderle, D; Série Harmônica. Disponivel em: <http://www.dirsom.com.br/index_htm_files/ Serie>. Acesso em: 13 de outubro de 2019.

[2] Benjamin, A. T; Proofs that Really Count: The Art of Combinatorial Proof. MAA, Washington, 2002.

[3] Brasil. Ministério da Educação; Base Nacional Comum Curricular. Brasília, 2018.

[4] Benjamin, A. T; A Stirling Encounter with Harmonic Numbers. Mathematics Magazine, 2002. Disponível em: <https://www.math.hmc.edu/ benjamin/papers/harmonic.pdf>. Acesso em: 13 de outubro de 2019.

[5] Garcia, A; Lequain, Y.; Elementos de Álgebra. Impa, Rio de Janeiro, 2008.

[6] Silva, N. A; Os números de Stilring. UFGD, Dourados, 2018.

[7] Pereira, M. C; Matemática e Música: De Pitágoras aos dias de hoje. UniRio, Rio de Janeiro, 2013. Disponivel em: <http://www2.unirio.br/unirio/ccet/profmat/tcc/2011/tcc-marcos>. Acesso em: 13 de outubro de 2019.

[8] Santos, J. P. O; Mello, M. P; Murari, I. T. C; Introdução à Análise Combinatória. Ciência Moderna, Rio de Janeiro, $200 \%$.

[9] Stewart, J.; Cálculo. Volume 2. Cengarge Learning, São Paulo, 2013.

[10] Permutações circulares e os números de Stirling do primeiro tipo; Clubes de Matemática da Obmep, 2020. Disponível em: <http://clubes.obmep.org.br/blog/ sala-de-estudo-permutacoes-circulares-e-os-numeros-de-stirling-do-primeiro-tipo/>. Acesso em: 19 de novembro de 2020.

Gabriel F. Pinheiro

Universidade Federal da Grande Dourados $<$ freitasgabriel688@gmail.com>

Irene M. Craveiro

Universidade Federal da Grande Dourados <irenecraveiro@ufgd.edu.br>

Naiguiel A. da Silva Escola Estadual <naiguiel_20@hotmail.com>

Recebido: 23/11/2019

Publicado: 21/12/2020 